\title{
Performance of Space-Division Multiplexed Systems Adopting Multi-Core Fibers and Receiver Diversity
}

\author{
Mai A. F. Banawan', Amira M. Hussein ${ }^{1}$, Ziad A. El-Sahn' ${ }^{1}$, and Hossam M. H. Shalaby ${ }^{1,2}$ \\ ${ }^{1}$ Photonics Group, Electrical Engineering Department, Alexandria University, Alexandria 21544, Egypt \\ Email : mai.a.f.banawan@gmail.com, amira.hussein13@gmail.com, ziad.elsahn@ieee.org \\ ${ }^{2}$ Department of Electronics and Communications Engineering Egypt-Japan University of Science and Technology \\ Alexandria 21934, Egypt. Email: shalaby@ieee.org
}

\begin{abstract}
The performance of MCF-based systems with MRC receiver diversity is studied at different levels of inter-core crosstalk. We demonstrate improvement in system performance at high values of crosstalk without the need for extensive MIMO-DSP.

OCIS codes: (060.2330); (060.2360);(060.4230)
\end{abstract}

\section{Introduction}

Space-division multiplexing (SDM) based on multi-core fibers (MCFs) is a promising technology to overcome the capacity limit of single mode fibers (SMFs) [1]. However, inter-core crosstalk (XT), arising from coupling between cores, limits the performance over long distances. One can design low-count MCF with low inter-core XT. Alternatively, to increase the capacity, it is necessary to use high-count MCFs which definitely require multipleinput multiple-output digital signal processing (MIMO-DSP) to counteract XT. The number of equalizers required for optimal MIMO detection grows quadratically with number of cores, also the equalizer length should ensure compensation of differential group delay (DGD) between cores. Hence, the required MIMO-DSP computational complexity is potentially high [2].

Recently, MCF-based systems with receiver diversity using maximum ratio combining (MRC) have been proposed to mitigate fiber nonlinear impairments [3]. The Q-factor is enhanced by 2 and $4 \mathrm{~dB}$ using 2 and 4 diversity cores, respectively. Thus, higher signal-to-noise ratio (SNR) and larger transmission distances could be achieved. Although it was demonstrated that increasing the number of diversity cores, i.e., the diversity order M, improves the system performance, the effect of inter-core XT was not considered.

In this paper, we demonstrate MRC diversity through weakly-coupled MCF by considering a more realistic scenario, where MCF inter-core XT imposes performance limitations. In this work, we propose to extend the MCF model beyond four cores. Our simulations focus on 2, 4 and 8 diversity cores and higher number of cores can be considered. Also, we evaluate the improvement in Q-factor and transmission distance achieved by our proposed system even at $-10 \mathrm{~dB}$ of accumulated inter-core XT. We also show that high levels of XT can still be tolerated using higher order of MRC diversity, without the need of complicated MIMO-DSP.

\section{MCF-based systems with MRC diversity}

Fig. 1 (a) shows the proposed MCF-based system adopting MRC receiver diversity. At the transmitter side, the modulated optical signal is equally divided among M cores of a MCF. For effective combining, different copies of the signal are decorrelated with delays of sufficient bit periods using few meters of SMF before being introduced to the MCF link. At the receiver side, coherent detected signals from different cores are passed to a DSP block, where
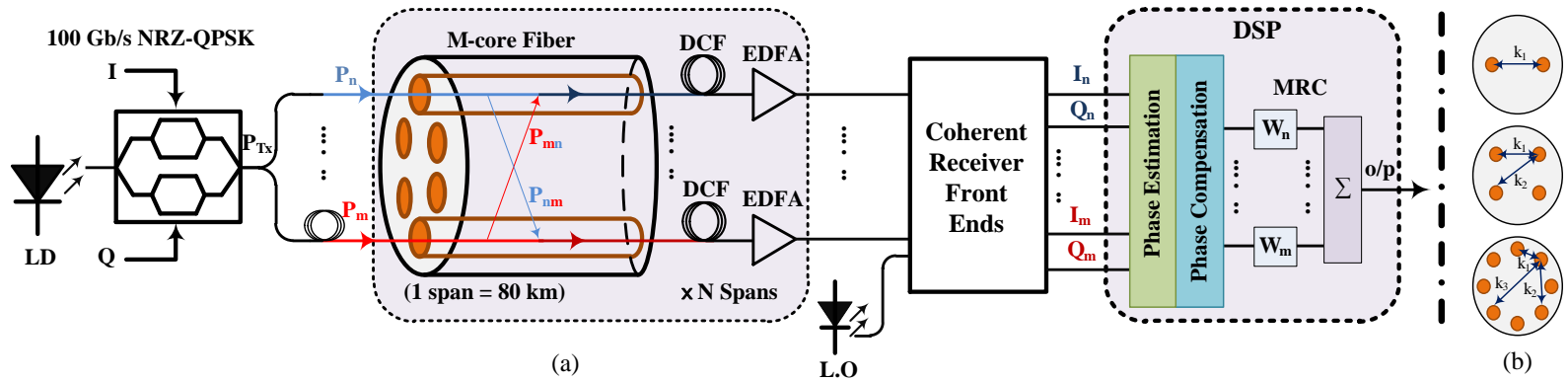

Fig. 1 (a) MCF-based system with MRC receiver diversity, (b) MCF for 2, 4 and 8-cores (DCF: dispersion compensating fiber, EDFA: erbiumdoped fiber amplifier, k: coupling coefficient, LD: laser diode, L.O: local oscillator, NRZ-QPSK: non-return-to-zero quadrature phase shift keying). Parameters: fiber attenuation $=0.2 \mathrm{~dB} / \mathrm{km}$, chromatic dispersion $=17 \mathrm{ps} / \mathrm{nm} / \mathrm{km}$, DGD $=0 \mathrm{ps}$, nonlinear refractive index $=3.1 \times 10^{-20}$ $\mathrm{m}^{2} / \mathrm{w}$ and effective area $=72 \mu \mathrm{m}^{2}$. EDFA Noise figure $=5 \mathrm{~dB}$. 
phase estimation and compensation are performed to ensure adding signals up coherently at the MRC combiner. In order to maximize the signal-to-noise ratio (SNR), all diversity branches are multiplied by a weight that is inversely proportional to the branch noise power, before summing them to perform MRC [3].

Inter-core XT effect is introduced by modeling the MCF using conventional coupled mode equations (CMEs) as in [4]. The XT introduces discrete changes in power levels caused by coupling between cores.

$$
\frac{d A_{m}}{d z}=-j \sum_{n \neq m} k_{m n} A_{n}(z) \exp \left(j \Delta \beta_{m n} z\right)
$$

where A represents the amplitude of the electric field, $\mathrm{k}_{\mathrm{mn}}$ is the mode-coupling coefficient from Core $\mathrm{n}$ to Core $\mathrm{m}$, the value of $\mathrm{k}_{\mathrm{mn}}$ varies between adjacent and non-adjacent cores and $\Delta \beta_{\mathrm{mn}}$ is the difference in the propagation constant between two cores, which includes two terms one of them is constant due to intrinsic propagation constant difference and the other is random due to random perturbations. Consequently, we model the MCF using cascaded SMF segments, where each segment is characterized by a random variation in the propagation constant that captures the effect of bending and twisting. Then the effect of accumulated XT through each segment is added by solving the CMEs analytically within each segment. Also, the DGD can be modeled by introducing a delay between signals at the end of each segment.

\section{System performance and simulation results}

The performance of the proposed system in Fig. 1(a) is evaluated via a MATLAB/OptiSystem co-simulation. A $100 \mathrm{~Gb} / \mathrm{s}$ NRZ-QPSK signal is generated and equally divided among identical cores of a homogeneous $1040 \mathrm{~km}$ MCF link composed of 13 spans. Each span consists of $80 \mathrm{~km}$ of MCF that is fully compensated by a DCF and a conventional EDFA per core. Each MCF span is modeled by 8 segments of SMF.

Fig. 2 (a) shows performance improvement in case of 2, 4 and 8 cores MCFs (shown in Fig. 1 (b)) with no XT compared to a reference SMF case that mimics the system without MRC diversity. It is obvious that MRC enhances the system performance compared to SMF. This is because that the total launched power $\mathrm{P}_{\mathrm{Tx}}$ is divided between the cores, which reducing the peak power and the fiber nonlinear effects. Thus, the Q-factor is improved by 2.5 and $5 \mathrm{~dB}$ for 2 and 4-cores diversity in agreement with results in [3], while for 8-cores an additional $1 \mathrm{~dB}$ (compared to 4-cores) is achieved. As a result, the Q-factor does not increase linearly with the diversity order M. So, balance between spatial diversity and spatial multiplexing (i.e., capacity) should be respected for better spatial utilization efficiency. The system performance under the influence of inter-core XT is illustrated in Fig. 2 (b) for the case of 4cores diversity. Compared to the case without XT, the Q-factor degrades by $0.5,1.5$ and $3.5 \mathrm{~dB}$ when increasing the XT levels to $-50,45$ and $-40 \mathrm{~dB} / \mathrm{km}$, respectively. Although inter-core XT imposes limitations, the four cores diversity system still has about $1.5 \mathrm{~dB}$ improvement than SMF.

The maximum achievable reach is shown in Fig. 3(a), it is calculated at the FEC threshold (BER $=10^{-3}$ ). The figure shows that SMF maximum reach is limited to $940 \mathrm{~km}$, while maximum transmission reach for 4-cores diversity increases significantly up to $2330 \mathrm{~km}$ without XT. Despite the fact that the $-40 \mathrm{~dB} / \mathrm{km}$ XT limits the maximum achievable reach to only $1110 \mathrm{~km}$, still it shows a better performance than SMF. The improvement in reach compared to SMF for different number of cores is summarized in Table 1, where 8-cores diversity exhibits the largest transmission distance.

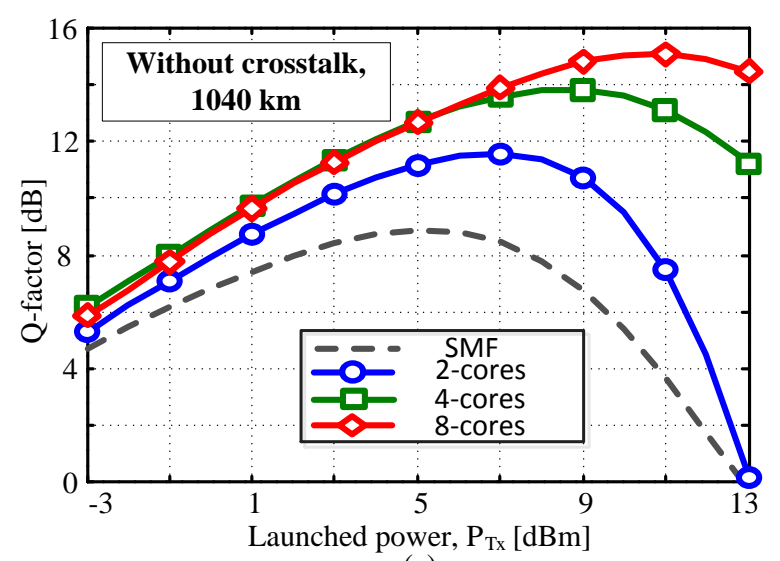

(a)

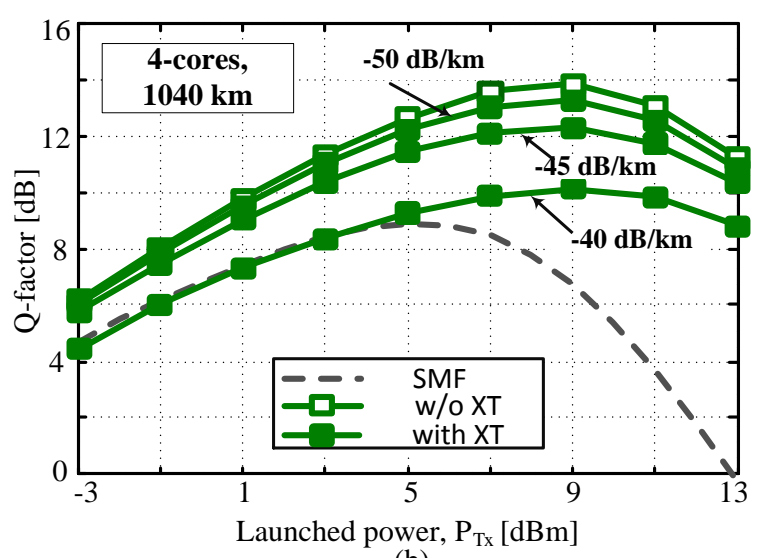

(b)

Fig. 2 (a) Q-factor versus launched power $\mathrm{P}_{\mathrm{Tx}}$ for 2, 4 and 8 cores in MCF, (b) Q-factor versus launched power $\mathrm{P}_{\mathrm{Tx}}$ for -50 , -45 and $-40 \mathrm{~dB} / \mathrm{km}$ crosstalk levels 


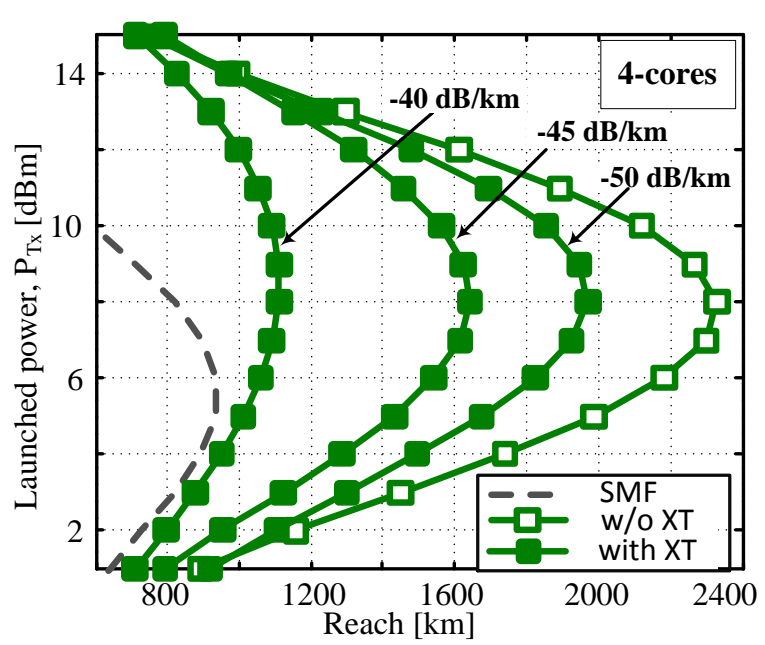

(a)

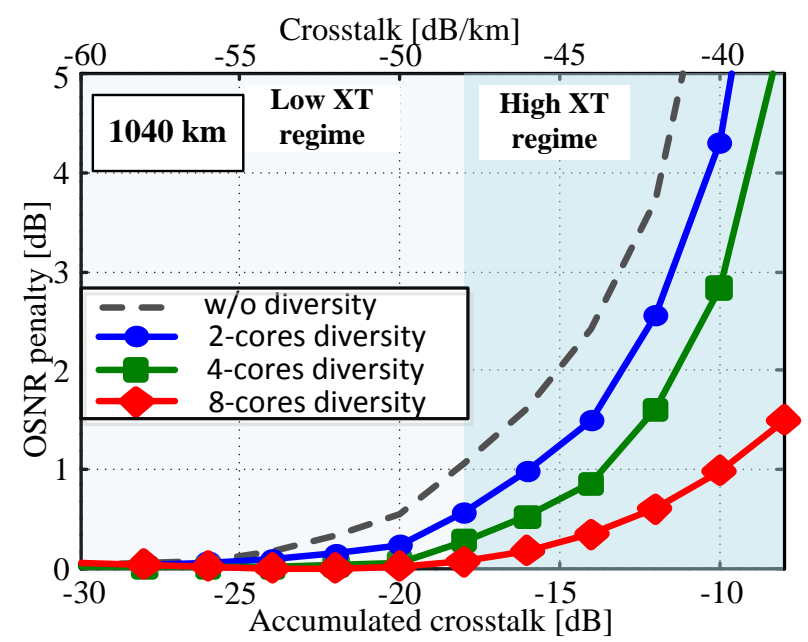

(b)

Fig. 3 (a) launched power $\mathrm{P}_{\mathrm{Tx}}$ versus reach for $-50,-45$ and $-40 \mathrm{~dB} / \mathrm{km}$ crosstalk levels, Forward error correction (FEC) Q-factor threshold of 9.42 $\mathrm{dB}$, which corresponds to bit error rate $(\mathrm{BER})=10^{-3}$, (b) OSNR penalty versus crosstalk for different MCFs at $0.1 \mathrm{~nm}$ resolution bandwidth, $\mathrm{P}_{\mathrm{Tx}}$ is taken at the optimum value of Q-factor.

Fig. 3 (b) represents the XT penalty compared to the system with no XT in terms of the optical SNR (OSNR) required to achieve $10^{-3} \mathrm{BER}$. MCF without diversity has two highlighted zones at $1 \mathrm{~dB}$ OSNR penalty. In low XT regime, it can support about $-18 \mathrm{~dB}$ XT level accumulated over $1040 \mathrm{~km}$ transmission link without MIMO-DSP in agreement with results in [5], while MIMO-DSP is required in the high XT regime. Diversity system can support $16,-14$ and $-10 \mathrm{~dB}$ total XT for 2, 4 and 8 cores, respectively. 8-cores diversity can tolerate the impact of higher XT levels more, thus tolerable end-to-end XT increases by increasing the diversity order $\mathrm{M}$ without the need for complex MIMO-DSP. Therefore scaling up the system capacity can be achieved using high-count MCFs adopting spatial multiplexing and a certain level of diversity that guarantees performance improvement as in Fig. 2 (b).

Table 1. Reach improvement

\begin{tabular}{ccccc}
\hline \multirow{2}{*}{$\mathbf{M}$} & \multicolumn{4}{c}{ Reach Improvement $[\mathbf{k m}]$ (over 940 $\mathbf{~ k m ~ o f ~ S M F ) ~}$} \\
\cline { 2 - 5 } & No XT & $\mathbf{X T} \mathbf{~ : ~} \mathbf{5 0}[\mathbf{d B} / \mathbf{k m}]$ & $\mathbf{- 4 5}[\mathbf{d B} / \mathbf{k m}]$ & $\mathbf{- 4 0}[\mathbf{d B} / \mathbf{k m}]$ \\
\hline $\mathbf{2}$ & 740 & 580 & 340 & 20 \\
$\mathbf{4}$ & 1390 & 1030 & 700 & 170 \\
$\mathbf{8}$ & 2020 & 1780 & 1300 & 820 \\
\hline
\end{tabular}

\section{Conclusion}

The performance of MCF-based system adopting MRC diversity shows that: Q-factor is improved with increasing diversity order at lower and certain higher levels of crosstalk compared to SMF. A balance between diversity and spatial multiplexing is needed to improve the system performance without the need of complex MIMO-DSP system. Furthermore the transmission distance can be extended when using MRC diversity compared to SMF. Hence, MCFbased MRC diversity can be considered as a promising candidate for long MCF transmission systems.

\section{References}

[1] D. J. Richardson, J. M. Fini \& L. E. Nelson, "Space-division multiplexing in optical fibers," Nat. Photonics. 7(5), (2013).

[2] Ry f, R., et al. "MIMO-Based Crosstalk Suppression in Spatially Multiplexed 3 x 56-Gb/s PDM-QPSK Signals for Strongly Coupled ThreeCore Fiber." IEEE Photonics Technology Letters 20.23 (2011).

[3] M. Koga, A. Mizutori \& T. Iida, "Optical diversity transmission and maximum-ratio combined receiver in multi-core fiber to mitigate fiber non-linear phenomenon”. IEICE Communications Express, 2(2), (2013).

[4] T. Hayashi, T. Taru, O. Shimakawa, T. Sasaki \& E. Sasaoka, "Design and fabrication of ultra-low crosstalk and low-loss multi-core fiber," Opt. Express 19(17), (2011).

[5] P. Winzer et al., "Penalties from In-Band Crosstalk for Advanced. Optical Modulation Formats" ECOC Tu.5.B.7 (2011). 